\title{
Role of Aldosterone in the Remnant Kidney Model in the Rat
}

\author{
E.L. Greene, S. Kren, and T.H. Hostetter \\ Department of Medicine, Renal Division, University of Minnesota, Minneapolis, Minnesota 55455
}

\begin{abstract}
The renin-angiotensin-aldosterone system (RAAS) participates in the injury sustained by the remnant kidney. Our studies assessed the importance of aldosterone in that model and the response of aldosterone to drugs interfering with the RAAS. Initially, four groups of rats were studied: SHAM-operated rats, untreated remnant rats (REM), REM rats treated with losartan and enalapril (REM AIIA), and REM AIIA rats infused with exogenous aldosterone (REM AIIA + ALDO). The last group was maintained with aldosterone levels comparable to those in untreated REM rats by constant infusion of exogenous aldosterone. REM rats had larger adrenal glands and $\mathrm{a}>10$-fold elevation in plasma aldosterone compared to SHAM. REM AIIA rats demonstrated significant suppression of the hyperaldosteronism as well as marked attenuation of proteinuria, hypertension, and glomerulosclerosis compared to REM. REM AIIA + ALDO rats manifested greater proteinuria, hypertension, and glomerulosclerosis than REM AIIA rats. Indeed, by 4 wk of observation all of these features of the experimental disease were similar in magnitude in REM AIIA + ALDO and untreated REM. In separate REM rats spironolactone administration did not reduce glomerular sclerosis but did transiently reduce proteinuria, lowered arterial pressure, and lessened cardiac hypertrophy. In summary, aldosterone contributes to hypertension and renal injury in the remnant kidney model. (J. Clin. Invest. 1996. 98:1063-1068.) Key words: aldosterone $\cdot$ renal failure glomerulus $\bullet$ hypertension $•$ renin
\end{abstract}

\section{Introduction}

Interruption of the renin angiotensin-aldosterone system (RAAS $)^{1}$ by converting enzyme inhibition or angiotensin II (Ang II) receptor antagonism dramatically alters the course of renal disease in the remnant kidney model (1-3). Further-

\footnotetext{
Address correspondence to Thomas H. Hostetter, University of Minnesota, Medicine-Renal Division, Box 736 UMHC, Minneapolis, MN 55455. Phone: 612-624-6917; FAX: 612-626-3840; E-mail: hoste002@ maroon.tc.umn.edu. E.L. Greene's current address is Nephrology Division, Medical University of South Carolina, Charleston, SC 29425.

Received for publication 24 February 1995 and accepted in revised form 13 May 1996.
}

1. Abbreviations used in this paper: Ang II, angiotensin II; RAAS, renin-angiotensin-aldosterone-system; REM, remnant; REM AIIA, REM rats treated with losartan and enalapril; REM AIIA + ALDO, REM AIIA rats infused with aldosterone.

J. Clin. Invest.

(C) The American Society for Clinical Investigation, Inc. 0021-9738/96/08/1063/06 \$2.00

Volume 98, Number 4, August 1996, 1063-1068 more, converting enzyme inhibition has proven clinically effective in slowing the decline in renal function of diabetic nephropathy (4). Reductions of systemic arterial pressure and glomerular pressure have generally been associated with these effects (13). Actions of Ang II in the kidney have been posited as the major mechanisms for maintenance of elevated glomerular pressure and reductions in glomerular pressure have been attributed, at least in part, to removal of the intrarenal effects of Ang II (1-3). Other nonhemodynamic but intrarenal actions of Ang II such as growth promoting effects and enhancement of ammoniagenesis may also contribute to progressive renal injury and relief from such actions may thereby reduce injury (1). However, the participation of circulating aldosterone in chronic glomerular damage and the possibility that the beneficial effects of RAAS blockade derive from aldosterone suppression have not been systematically examined. We report experiments designed to test the role of aldosterone in the remnant kidney model of progressive renal disease.

\section{Methods}

Study 1. Adult male Sprague-Dawley rats weighing 200-300 grams were subjected to sham (SHAM) renal ablation consisting of laparotomy and manipulation of the renal pedicles or subtotal renal ablation to produce the remnant kidney model (REM). Ablation consisted of right nephrectomy and infarction of a portion of the left kidney by ligation of one segmental renal artery. $3 \mathrm{~d}$ after reduction in renal mass, the rats with remnant kidneys had creatinines measured on tail vein blood. They were stratified on the basis of that measurement and assigned to one of three groups such that initial serum creatinines were equivalent in all REM groups. The first group with partial ablation consisted of rats with remnant kidneys and no other manipulation (REM). The second partially ablated group consisted of rats that were placed on a combination of enalapril and losartan in their drinking water (50 and $180 \mathrm{mg} /$ liter, respectively) (REM AIIA). Both agents were used simultaneously with the plan of maximally inhibiting both production and action of Ang II. The third group of partially ablated animals (REM AIIA + ALDO) were treated similarly with losartan and enalapril but were subcutaneously infused with aldosterone by osmotic mini-pump at either 30 or $40 \mu \mathrm{g} / \mathrm{kg}$ per $\mathrm{d}$. The rates of aldosterone infusion were determined by initially measuring plasma aldosterone in subgroups of SHAM, REM, and REM AIIA rats. Then, based on the data of Martin et al. and several preliminary trials with differing rates of infusion, these two rates were chosen as likely to bracket the level of aldosterone in untreated REM rats (5). These two rates yielded plasma aldosterone levels that were not significantly different from one another. For the $30 \mu \mathrm{g} / \mathrm{kg}$ per d infusion, plasma aldosterone averaged $504 \pm 139 \mathrm{pg} / \mathrm{ml}(n=7)$. For the $40 \mu \mathrm{g} /$ $\mathrm{kg}$ per $\mathrm{d}$ infusion, plasma aldosterone averaged $468 \pm 87 \mathrm{pg} / \mathrm{ml}(n=$ 6). Also systolic pressure and proteinuria were similar at these two rates of aldosterone infusion. The data for the two levels of aldosterone administration were, therefore, pooled into a single group (REM AIIA + ALDO) for the 2-wk study. Small numbers of deaths occurred in various groups; animals that died before the completion of the study were not included in any of the analyses. All rats were allowed free access to water and standard rat chow (protein $24 \%$ and sodium 0.44\%; Teklad Premier Laboratory Diets, Madison, WI). 12-14 d after assignment to groups, the rats were placed in metabolic cages for collection of urine for $24 \mathrm{~h}$. Systolic blood pressures were mea- 
sured in the awake state by the tail cuff method. Rats were then returned to their standard housing for $1 \mathrm{~d}$ and killed on the following day by decapitation. Trunk blood was obtained for biochemical analyses and organs were removed for biochemical analyses and weighing. Adrenal weights are expressed as the sum of both glands.

Study 2. A second cohort of rats comprising the same four groups (except that the infusion of aldosterone was at $35 \mu \mathrm{g} / \mathrm{kg}$ per $\mathrm{d}$ in the REM AIIA + ALDO group) was maintained for 4 wk. Measurement of systolic arterial pressure, plasma potassium, and urine collections were obtained on these rats after 2 and 4 wk of assignment to groups. At 4 wk under methohexital anesthesia, their kidneys were perfusion fixed with formaldehyde, embedded in paraffin, sectioned, and stained with hematoxylin and eosin. The prevalence of glomerular sclerosis was determined on these sections in a blinded manner. For each rat, at least 100 glomerular profiles were assessed for the presence or absence of any sclerosis. The results are expressed as the percentage of glomeruli having any sclerotic component.

Study 3. A third cohort of rats made up of two groups (both REM) was maintained for $4 \mathrm{wk}$. These rats were fed the same chow as those of the other two cohorts but in powdered form. One group had spironolactone added to the chow at $5.56 \mathrm{grams} / \mathrm{kg}$. These two groups of rats were studied in the same fashion as in study 2 for $4 \mathrm{wk}$ with the degree of glomerulosclerosis determined at the conclusion of the experiment.

The drug was administered orally in the food because preliminary experiments demonstrated that spironolactone is insoluble in water and its subcutaneous administration as an aqueous suspension led to precipitate, apparently of the drug, within the subcutaneous tissue. Also spironolactone solubility in propylene glycol was excellent but when administered in this solvent, sterile inflammatory lesions developed. The dose of spironolactone was chosen by preliminary studies using the mineralocorticoid salt model of hypertension. Rats underwent unilateral nephrectomy alone or unilateral nephrectomy with normal saline as drinking water, and continuous infusion of aldosterone $50 \mu \mathrm{g} / \mathrm{kg}$ per $\mathrm{d}$ by Alzet pump. Spironolactone, in the powdered food, at 5.56 grams $/ \mathrm{kg}$, reduced the increment in systolic pressure by $75 \%$. However, spironolactone at $1.39 \mathrm{grams} / \mathrm{kg}$ did not influence pressure. These doses were designed to yield $\sim 400 \mathrm{mg} / \mathrm{d}$ per $\mathrm{kg}$ rat weight and $100 \mathrm{mg} / \mathrm{d}$ per $\mathrm{kg}$ rat weight, respectively, assuming 18 grams of daily food intake by rats weighed 250 grams.

Plasma renin activity and plasma aldosterone were determined by radioimmunoassay. For renin activity, the generation of angiotensin I was measured using a kit from New England Nuclear (Boston, MA). For aldosterone, a kit manufactured by Diagnostic Products (Los Angeles, CA) was used. Adrenal renin concentration was determined as described by Baba et al. but using the same radioimmunoassay for renin activity as used for plasma (6). The protein content of the adrenals and urine was measured by the Coomassie dye method.

Statistical analysis was performed using ANOVA with statistical significance determined by the Bonferroni modification of the $t$ test for multiple comparisons. The results are expressed as means \pm standard deviations.

\section{Results}

Study 1. Body weight was least in the animals in the REM group, an effect not altered by AIIA (Table I). The REM AIIA + ALDO group achieved body weights intermediate between REM and SHAM and which were statistically different from both. However, the weight gain over the 2 wk after assignment to the groups did not differ among the three groups with remnant kidneys but was less than in the SHAM group.

The plasma aldosterone level was increased $>10$-fold in REM as compared to SHAM (526 \pm 50 vs $50 \pm 12 \mathrm{pg} / \mathrm{ml}$ ) (Table I). This increase in plasma aldosterone in the REM group was largely blocked with RAAS blockade in the REM AIIA group such that in this latter group, plasma aldosterone was reduced to $181 \pm 124 \mathrm{pg} / \mathrm{ml}$. The infusion of exogenous aldosterone with ongoing RAAS interruption was designed to elevate plasma levels into the range of those seen in the REM group. The mean value of plasma aldosterone was $487 \pm 114 \mathrm{pg} / \mathrm{ml}$ in the REM AIIA + ALDO group, a value significantly greater than that of the SHAM group, but not significantly different from the value of $526 \pm 250 \mathrm{pg} / \mathrm{ml}$ in the untreated REM group. Consistent with the hyperaldosteronism, adrenal weights were increased in REM as compared to SHAM (63 \pm 4 vs $51 \pm 4 \mathrm{mg}$ ). This adrenal hypertrophy was partially blocked in the REM AIIA group, but the REM AIIA + ALDO group had an intermediate value not significantly different from any of the other three groups. As expected, plasma renin activity was elevated in the REM AIIA group compared with SHAM and the untreated REM group. However, exogenous aldosterone, in the AIIA + ALDO group, suppressed plasma renin activity to a level indistinguishable from that in SHAM and untreated REM groups. Finally, adrenal renin concentration tended to be suppressed in the REM group compared with SHAM and

Table I. Data from Study 1 at End of 2 Wk

\begin{tabular}{|c|c|c|c|c|c|c|c|c|c|}
\hline & $\begin{array}{c}\text { Final } \\
\text { body weight }\end{array}$ & $\begin{array}{c}\text { Body } \\
\text { weight gain }\end{array}$ & $\begin{array}{c}\text { Left } \\
\text { kidney weight }\end{array}$ & SBP & $\mathrm{U}_{\text {PROT }} \mathrm{V}$ & $\mathrm{P}_{\mathrm{ALDO}}$ & $\begin{array}{l}\text { Adrenal } \\
\text { weight }\end{array}$ & PRA & Adrenal RC \\
\hline & grams & grams & grams & $m m ~ H g$ & $m g / 24 h$ & $\mathrm{pg} / \mathrm{ml}$ & $m g$ & $n g / m l$ per $h$ & $n g / m g$ per $h$ \\
\hline SHAM & 336 & 84 & 1.11 & 118 & 10 & 50 & 51 & 3.1 & 2.2 \\
\hline$(n=6$ or 7$)$ & \pm 16 & 12 & 0.06 & 9 & 2 & 12 & 4 & 0.7 & 1.2 \\
\hline REM & $277 *$ & 66 & 1.37 & $185^{*}$ & $121 *$ & $526 *$ & $63^{*}$ & 4.3 & 1.3 \\
\hline$(n=11-25)$ & 27 & 26 & 0.23 & 26 & 54 & 250 & 4 & 2.08 & 0.8 \\
\hline REM AIIA & $285^{*}$ & 74 & 1.16 & $125^{\ddagger}$ & $31^{\ddagger}$ & $181^{\ddagger}$ & $56^{\ddagger}$ & $13.3^{\ddagger}$ & 3.6 \\
\hline$(n=5-10)$ & 15 & 17 & 0.25 & 14 & 12 & 124 & 4 & 12.3 & 2.4 \\
\hline \multicolumn{10}{|l|}{ REM AIIA } \\
\hline + ALDO & $302 * \ddagger$ & $66^{*}$ & $1.49 * \S$ & $149 * \$ \S$ & $87 * \S$ & $487 * \S$ & 60 & 5.2 & $0.50 * \S$ \\
\hline$(n=9$ or 13$)$ & 14 & 11 & 0.23 & 21 & 28 & 114 & 7 & 5.0 & 0.20 \\
\hline
\end{tabular}

Values are means \pm standard deviations. ${ }^{*} P<0.05$ vs SHAM; ${ }^{\ddagger} P<0.05$ vs REM; ${ }^{\S} P<0.05$ vs REM AIIA. SBP, conscious systolic blood pressure; $\mathrm{U}_{\mathrm{PROT}} \mathrm{V}$, protein excretion rate; $\mathrm{P}_{\mathrm{ALDO}}$, plasma aldosterone level; PRA, plasma renin activity; Adrenal RC, adrenal renin concentration. 
Table II. Data from Study 2 at End of 4 wk

\begin{tabular}{|c|c|c|c|c|c|c|c|c|c|}
\hline & $\begin{array}{c}\text { Final } \\
\text { body weight }\end{array}$ & $\begin{array}{c}\text { Body } \\
\text { weight gain }\end{array}$ & $\begin{array}{l}\text { Left kidney } \\
\text { weight }\end{array}$ & SBP & Heart weight & $\mathrm{U}_{\mathrm{PROT}} \mathrm{V}$ & $\mathrm{P}_{\mathrm{K}}$ & $\begin{array}{l}\text { Adrenal } \\
\text { weight }\end{array}$ & $\begin{array}{l}\text { Glomerular } \\
\text { sclerosis }\end{array}$ \\
\hline & grams & grams & grams & $m m H g$ & grams & $m g / 24 h$ & $m M$ & $m g$ & $\%$ \\
\hline SHAM & 347 & 99 & 1.19 & 118 & 1.03 & 19 & 5.0 & 46 & 1.9 \\
\hline$(n=6)$ & \pm 18 & 16 & 0.08 & 10 & 0.05 & 6 & 0.6 & 2 & 1.2 \\
\hline REM & 309 & 96 & $1.91 *$ & $212 *$ & $1.33^{*}$ & $203 *$ & 5.0 & $69^{*}$ & $37.2 *$ \\
\hline$(n=7)$ & 50 & 40 & 0.55 & 20 & 0.19 & 103 & 0.4 & 9 & 26.5 \\
\hline REM AIIA & 307 & 97 & $1.47^{*}$ & $109^{\ddagger}$ & $0.88^{* \neq}$ & $30^{\ddagger}$ & $6.2 * \neq$ & $57^{*}$ & $4.7^{\ddagger}$ \\
\hline$(n=7)$ & 25 & 20 & 0.18 & 11 & 0.11 & 15 & 0.4 & 4 & 5.2 \\
\hline \multicolumn{10}{|l|}{ REM AIIA } \\
\hline+ ALDO & 312 & 106 & $2.03^{*}$ & $186^{* \S}$ & $1.28 * \S$ & $217 * \S$ & $4.5^{\S}$ & $60^{*}$ & $25.3^{* \S}$ \\
\hline$(n=6)$ & 24 & 26 & 0.48 & 26 & 0.12 & 71 & 0.5 & 8 & 11.1 \\
\hline
\end{tabular}

Values are means \pm standard deviations. $* P<0.05$ vs SHAM, ${ }^{\ddagger} P<0.05$ vs REM, ${ }^{\S} P<0.05$ vs REM AIIA. $\mathrm{P}_{\mathrm{K}}$, plasma potassium level; SBP, conscious systolic blood pressure; $\mathrm{U}_{\mathrm{PROT}} \mathrm{V}$, protein excretion rate.

exaggerated in REM AIIA. The REM AIIA + ALDO group demonstrated suppression of adrenal renin activity when compared with SHAM and REM AIIA.

Systolic blood pressure was highest in the REM group at $185 \pm 26 \mathrm{mmHg}$. The systemic hypertension was essentially prevented in the REM AIIA group with blood pressures reduced to $125 \pm 14 \mathrm{mmHg}$. This effect of losartan and enalapril was in part reversed with aldosterone infusion in the REM AIIA + ALDO group, which developed a systolic blood pressure of $149 \pm 21 \mathrm{mmHg}$. This pattern of effects on systemic blood pressure was mirrored in the variation of proteinuria among the groups. The untreated REM group had $>10$-fold elevation of proteinuria compared with the SHAM group (121 \pm 54 vs $11 \pm 2 \mathrm{mg} / \mathrm{d}$ ). As with hypertension, proteinuria was nearly vitiated by enalapril and losartan in the REM AIIA group ( $33 \pm 13 \mathrm{mg} / \mathrm{d})$. Aldosterone infusion caused proteinuria of $87 \pm 28 \mathrm{mg} / \mathrm{d}$, a value significantly greater than both REM AIIA and SHAM. Thus, at 2 wk $\sim 1 / 3$ of the systemic hypertension and $2 / 3$ of the proteinuria present in REM, but obvi- ated in REM AIIA, was reproduced by aldosterone infusion in the REM AIIA + ALDO group.

Study 2. Body weights were similar among the three groups with the remnant kidneys while those in SHAM group had greater final body weights (Table II). However, by 4 wk the increment in body from the time of assignment to their groups was similar among all four groups.

As in the 2-wk study, adrenal weight was least in the SHAM group. Also, blockade of Ang II production and action again blunted this adrenal hypertrophy in the REM AIIA group. Plasma potassiums were within the normal range and similar in the SHAM, untreated REM, and REM AIIA + ALDO groups. However, in the REM AIIA group potassium levels were distinctly elevated.

The pattern of awake systolic blood pressure differences within this cohort of rats was similar at $2 \mathrm{wk}$ to that observed in the cohort used for the 2-wk study (Fig. 1, $A$ ). Specifically, the highest pressures were observed in untreated REM animals with restitution of a large fraction of the increment in arterial
A

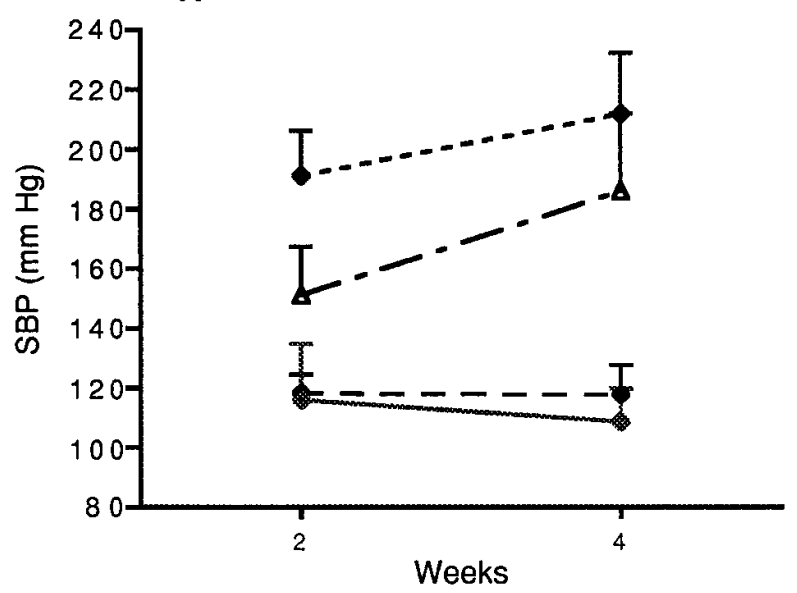

B

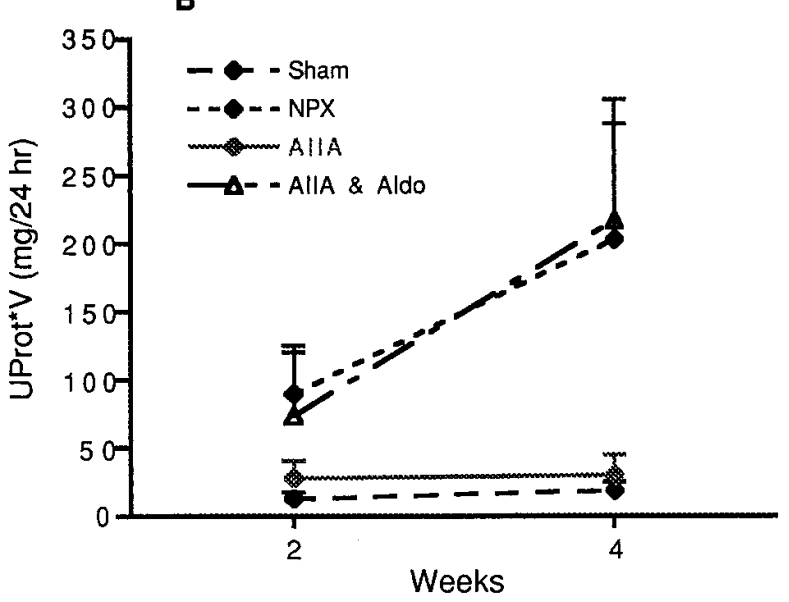

Figure 1. (A) Conscious systolic blood pressure, measured at 2 and $4 \mathrm{wk}$ in rats in the 4-wk study. (B) Urinary protein excretion at 2 and 4 wk in rats in the 4-wk study. Numerical values for the 4-wk data are provided in Table II. The error bars are standard errors of the means. 
Table III. Data from Study 3

\begin{tabular}{|c|c|c|c|c|c|c|c|c|c|c|c|c|}
\hline & $\begin{array}{c}\text { Final } \\
\text { body weight }\end{array}$ & $\begin{array}{c}\text { Body } \\
\text { weight gain }\end{array}$ & Food intake & $\begin{array}{l}\text { Left kidney } \\
\text { weight }\end{array}$ & \multicolumn{2}{|c|}{ SBP } & $\begin{array}{c}\text { Heart } \\
\text { weight }\end{array}$ & \multicolumn{2}{|c|}{$\mathrm{U}_{\mathrm{PROT}} \mathrm{V}$} & $P_{k}$ & $\begin{array}{c}\text { Adrenal } \\
\text { weight }\end{array}$ & $\begin{array}{l}\text { Glomerular } \\
\text { sclerosis }\end{array}$ \\
\hline & grams & grams & grams $/ 24 \mathrm{~h}$ & grams & \multicolumn{2}{|c|}{$\mathrm{mmHg}$} & grams & \multicolumn{2}{|c|}{$m g / 24 h$} & $m M$ & $m g$ & $\%$ \\
\hline & & & at $4 \mathrm{wk}$ & & at $2 \mathrm{wk}$ & at $4 \mathrm{wk}$ & & at $2 \mathrm{wk}$ & at $4 \mathrm{wk}$ & at $2 \mathrm{wk}$ & & \\
\hline REM & 315 & 59 & 19 & 1.96 & 201 & 208 & 1.33 & 201 & 283 & 5.3 & 71 & 31 \\
\hline$(n=8-19)$ & \pm 27 & 31 & 8 & 0.44 & 19 & 17 & 0.13 & 89 & 148 & 0.4 & 4 & 16 \\
\hline REM+ & 300 & 48 & 23 & 1.79 & 192 & $190 *$ & $1.21 *$ & $130^{*}$ & 243 & 5.2 & 73 & 26 \\
\hline $\begin{array}{l}\text { spironolactone } \\
(n=8-21)\end{array}$ & 31 & 31 & 6 & 0.35 & 20 & 28 & 0.10 & 54 & 74 & 0.4 & 8 & 11 \\
\hline
\end{tabular}

Values are means \pm standard deviation. ${ }^{*} P<0.05$ vs REM. Abbreviations are same as in Table II.

pressure by exogenous aldosterone infusion in the REM AIIA + ALDO group. This pattern persisted in the measurements made at 4 wk by which point an even larger fraction of the increment in blood pressure sustained in the untreated REM group was reproduced by aldosterone infusion in the REM + ALDO group. As with blood pressure, the patterns of proteinuria observed in the 2 -wk study were essentially reproduced at $2 \mathrm{wk}$ in this cohort (Fig. 1, B). This pattern of proteinuria progressed such that by 4 wk essentially identical levels of proteinuria were observed in the REM AIIA + ALDO group as in the untreated REM group. Proteinuria continued to be almost completely prevented in the REM AIIA group even by 4 wk.

Glomerular sclerosis was greatest in the untreated REM

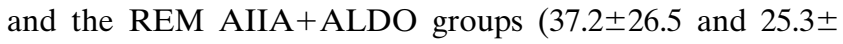
$11.1 \%$, respectively). Thus, aldosterone infusion despite ongoing enalapril and losartan administration reproduced most of the glomerulosclerosis seen in the untreated REM group. As expected, SHAM animals and those remnant animals in the REM AIIA group manifested similar prevalences of sclerotic glomeruli (1.9 \pm 1.2 and $4.7 \pm 5.2 \%$, respectively), which were substantially lower than seen in the other two groups.

Study 3. In the rats studied for the effects of spironolactone (see Table III), neither body weight nor food intake was altered by spironolactone intake. The average dose of spironolactone was $420 \mathrm{mg} / \mathrm{d}$ per $\mathrm{kg}$ of rat weight as measured on the last day. Plasma potassium and adrenal weights were not affected by the drug. However, it reduced systolic arterial pressure at 4 wk by $18 \mathrm{mmHg}$. Heart weight was also lower with spironolactone therapy. The drug diminished proteinuria by $\sim 40 \%$ at 2 wk but this was not statistically significant by 4 wk. Likewise, at $4 \mathrm{wk}$, there was no detectable difference in the degree of glomerulosclerosis with spironolactone treatment.

\section{Discussion}

Our studies demonstrate that adrenal hypertrophy and hyperaldosteronism attend the hypertension, proteinuria, and glomerulosclerosis characteristic of the remnant kidney model. The adrenal enlargement has been previously reported, but the elevated plasma aldosterone has not been previously commented upon in this experimental disease (7). As expected, combined therapy with the Ang II receptor blocker, losartan, and the converting enzyme inhibitor, enalapril, lessened the hypertension, proteinuria, and glomerulosclerosis of the remnant kid- ney (1-3). This regimen also attenuated the hyperaldosteronism. Most importantly, the reproduction of the hyperaldosteronism by exogenous steroid infusion on the background of the pharmacologic blocking maneuvers restored most of the arterial hypertension, proteinuria, and glomerulosclerosis observed with the untreated, subtotally ablated kidney.

Hyperaldosteronism has been noted as a component of clinical chronic renal insufficiency. Hené and colleagues described elevation in plasma aldosterone in patients with stable chronic renal insufficiency of various etiologies (8). In their cross-sectional analysis of subjects with a range of renal function, the level of aldosterone appeared to increase as creatinine clearance fell below $\sim 70 \mathrm{ml} / \mathrm{min}$, rising ultimately threeto fourfold over the values measured above that clearance. Other clinical investigations have also identified heightened aldosterone levels in renal insufficiency. For example, Berl et al. studied eight subjects, whose average creatinine clearance was $14 \mathrm{ml} / \mathrm{min}$, and five of them had plasma aldosterone levels above the normal range (9). Likewise nine subjects with even better renal function (average inulin clearance $=27 \mathrm{ml} / \mathrm{min}$ ), who were studied by Reams and Bauer, had an average plasma aldosterone level more than fourfold greater than normal values $(10,11)$. The significance of this hyperaldosteronism to progression has not been much mooted but Walker did note a significant correlation between aldosterone level and rate of renal decay in a longitudinal study of patients with diabetes (12). While elevated plasma aldosterone levels have been remarked upon in clinical renal impairment, hyperaldosteronism has not been a recognized feature of the remnant kidney model. Adrenal hypertrophy had been previously linked to this experimental model. Specifically, Morrison documented increased adrenal weight after subtotal ablation and in qualitatively evaluating this growth he reported that the zona glomerulosa was more prominently widened than the reticulata (7). Thus, our studies extend this latter observation and demonstrate that, like the clinical condition, the remnant kidney model manifests an increased plasma aldosterone.

Pharmacologic interdiction of the RAAS strikingly mitigates the injury incurred by the untreated remnant kidney (1$3)$. Although the effect of this therapy on aldosterone had not been previously investigated, several other observations in this experimental model do suggest that aldosterone may contribute to its progressive injury. Quan and coworkers performed adrenalectomy in rats with subtotal nephrectomy (13). However, despite large doses of replacement glucocorticoid, sev- 
eral cardinal features of the disease including proteinuria, histologic measures of renal injury, and hypertension were still less than in similarly ablated rats with intact adrenal glands. The authors did not replace aldosterone having provided a high salt ration instead. But since mineralocorticoids were not maintained, we speculate that their absence accounted for the mitigation of renal disease after adrenalectomy even with glucocorticoid therapy. Of additional interest, heparin administration provides remarkably complete protection from injury in the remnant kidney model (14-16). Although this effect may emanate from any of several actions of heparin, suppression of aldosterone production should be considered as one of these potentially beneficial actions. Finally, our present data supply yet more direct evidence that aldosterone provokes the key elements of this experimental disease, since the infusion of this steroid alone largely reproduced the syndrome.

The effects of converting enzyme inhibition on the course of clinical renal insufficiency have been generally salutary (1, $4,10,18)$. Furthermore, aldosterone appears to respond to this therapy, although this effect has not been extensively examined. Bauer and Reams reported that the average baseline value of plasma aldosterone of $234 \mathrm{pg} / \mathrm{ml}$ in their subjects fell to $135 \mathrm{pg} / \mathrm{ml}$ after $1 \mathrm{mo}$ of enalapril administration (10). Ruilope and colleagues, also studying patients with renal insufficiency, observed a significant decline of plasma aldosterone from 266 to $105 \mathrm{pg} / \mathrm{ml}$ with 6 mo of captopril treatment (18). However, by $12 \mathrm{mo}$ this level had risen to $234 \mathrm{pg} / \mathrm{ml}$, a value insignificantly different from the baseline. That aldosterone secretion may "escape" from the suppressive actions of converting enzyme inhibitors has been noted in studies of these drugs in other clinical settings but the therapeutic consequences of such escape are uncertain (18). Moreover, the possibility that the beneficial actions of these agents in clinical renal insufficiency derive, at least in part, from a diminution in aldosterone levels has not been investigated.

Mineralocorticoids have been held responsible for scarring and injury in extrarenal components of the cardiovascular system. Weber and colleagues have adduced evidence that myocardial fibrosis can result from mineralocorticoid action, evidence recently confirmed by Young et al. $(19,20)$. These data also suggest that the fibrogenic and hypertrophic effects may not be solely a response to systemic hypertension engendered by these steroids. In the present study, the effects of spironolactone to lessen cardiac hypertrophy are also consistent with the findings of these investigators. Our data, however, suggest that aldosterone does contribute to the arterial hypertension of the remnant kidney model. But, the mechanism by which it does so is yet uncertain. Both sodium retention and vasoconstrictive effects of mineralocorticoids have been incriminated in the hypertension of the classic mineralocorticoid/salt model and both categories of action may be at play in partial renal ablation (21-23). We doubt, however, that salt retention is an entirely sufficient explanation for our findings in the subtotal ablation model. We hold this view based on previous studies both from our laboratory and those of others demonstrating virtually no influence on arterial hypertension of diets differing widely in sodium content $(14,24,25)$. Vascular actions of aldosterone, for example, to enhance ion permeability in vascular smooth muscle, to reset baroreceptors, or to amplify local vasoconstrictor systems likely ally in the hypertensive action of aldosterone both in the remnant kidney and the mineralocorticoid/salt model (26-29). Glomerular capillary pressures were not measured but we suspect are elevated in the aldosterone supplementation since they are clearly increased in the mineralocorticoid salt model of hypertension (30). Such an effect would contribute to injury.

Nonhemodynamic actions of aldosterone may also participate in its renal as well as cardiac fibrotic consequences. In vitro studies of cultured mesangial cells revealed increased type IV collagen production after incubation with aldosterone and if such an effect obtained in vivo, the potential consequences for glomerular matrix and basement membrane expansion are easily envisioned (31). Although the distal tubule is usually considered the target for aldosterone action in the kidney, transcripts for the mineralocorticoid receptor have been detected in the glomerulus, albeit at lower levels than in the distal tubular epithelium, and they might serve to mediate steroid actions at this site (32). Also, nongenomic effects of aldosterone mediated through membrane rather than nuclear receptors, although not yet examined for glomerular cells, might transduce direct renal responses outside of the distal tubule (33). Thus, both hypertensive and more direct cellular actions of aldosterone, including fibrogenic ones, may account for its sclerosing influence on the remnant glomerulus.

Administration of the mineralocortoid receptor blocker, spironolactone, had antihypertensive effects and a transient effect on proteinuria. It also mitigated cardiac hypertrophy. However, glomerular structural injury was not notably lessened. The reasons for these relatively modest effects, despite the demonstration of a sizable action of exogenous aldosterone in the rats with remnant kidneys receiving converting enzyme inhibition and angiotensin II receptor blockade, are not clear. Perhaps mineralocorticoid receptor blockade was incomplete or aldosterone may be acting through additional receptors such as the plasma membrane sites described by Wehling and colleagues (33). These latter membrane transducers' effects are not inhibitable by spironolactone analogs (34).

In summary, our studies demonstrate that hyperaldosteronism occurs in the remnant kidney model. Angiotensin converting enzyme inhibition and angiotensin II receptor antagonism reduced the elevated aldosterone levels and appeared to function, in large part, through this suppressive effect on aldosterone. Aldosterone inflicts much of the injury in the subtotal ablation model and may do so generally in chronic progressive renal disease.

\section{Acknowledgments}

We are grateful to Georgia Spiropoulos and Linda Chmielewski for expertly producing the manuscript.

The work was supported by National Institutes of Health Grant DK 31437 and a minority supplement to that grant.

\section{References}

1. Rosenberg, M.E., L.J. Smith, R. Correa-Rotter, and T.H. Hostetter. 1994. The paradox of the renin-angiotensin system in chronic renal disease. Kidney Int. 45:403-410.

2. Anderson, S., H.G. Rennke, and B.M. Brenner. 1986. Therapeutic advantage of converting enzyme inhibitors in arresting progressive renal disease associated with systemic hypertension in the rat. J. Clin. Invest. 77:1993-2000.

3. Lafayette, R.A., G. Mayer, S.K. Park, and T.W. Meyer. 1992. Angiotensin II receptor blockade limits glomerular injury in rats with reduced renal mass. J. Clin. Invest. 90:766-771.

4. Lewis, E.J., L.G. Hunsicker, R.P. Bain, and R.D. Rohde. 1993. The effect of angiotensin-converting-enzyme inhibition on diabetic nephropathy. $N$. Engl. J. Med. 329:1456-1462. 
5. Martin, R.S., W.J. Jones, and J.P. Hayslett. 1983. Animal model to study the effect of adrenal hormones on epithelial function. Kidney Int. 24:386-391.

6. Baba, K., Y. Doi, R. Franco-Saenz, and P.J. Mulrow. 1986. Mechanisms by which nephrectomy stimulates adrenal renin. Hypertension (Dallas). 8:9971002.

7. Morrison, A.B. 1962. Experimentally induced chronic renal insufficiency in the rat. Lab. Invest. 11:321-331.

8. Hené, R.J., P. Boer, H.A. Koomans, and E.J.D. Mees. 1982. Plasma aldosterone concentrations in chronic renal disease. Kidney Int. 21:98-101.

9. Berl, T., F.H. Katz, W.L. Henrich, A. de Torrente, and R.W. Schrier. 1978. Role of aldosterone in the control of sodium excretion in patients with advanced chronic renal failure. Kidney Int. 14:228-235.

10. Reams, G.P., and J.H. Bauer. 1986. Effect of enalapril in subjects with hypertension associated with moderate to severe renal dysfunction. Arch. Intern. Med. 146:2145-2148.

11. Williams, G.H., and R.G. Dluhy. 1987. Diseases of the adrenal cortex. In Harrison's Principles of Internal Medicine. 11th Edition. E. Braunwald, K.J. Isselbacher, R.G. Petersdorf, J.D. Wilson, J.D. Martin, and A.S. Fauci, editors. McGraw-Hill, New York. 1753-1775.

12. Walker, W.G. 1993. Hypertension-related renal injury: a major contributor to end-stage renal disease. Am. J. Kidney Dis. 22:164-173.

13. Quan, Z.Y., M. Walser, and G.S. Hill. 1992. Adrenalectomy ameliorates ablative nephropathy in the rat independently of corticosterone maintenance level. Kidney Int. 41:326-333.

14. Purkerson, M.L., P.E. Hoffsten, and S. Klahr. 1976. Pathogenesis of the glomerulopathy associated with renal infarction in rats. Kidney Int. 9:407-417.

15. Purkerson, M.L., D.M. Tollefsen, and S. Klahr. 1988. N-desulfated/ acetylated heparin ameliorates the progression of renal disease in rats with subtotal renal ablation. J. Clin. Invest. 81:69-74.

16. Olson, J.L. 1984. Role of heparin as a protective agent following reduction of renal mass. Kidney Int. 25:376-382.

17. Ruilope, L.M., B. Miranda, J.M. Morales, J.L. Rodicio, J.C. Romero, and L. Raij. 1989. Converting enzyme inhibition in chronic renal failure. Am. J. Kidney Dis. 13:120-126.

18. Pitt, B. 1995. "Escape" of aldosterone production in patients with left ventricular dysfunction treated with an angiotensin converting enzyme inhibitor: Implications for therapy. Cardiovas. Drugs Ther. 9:145-149.

19. Brilla, C.G., and K.T. Weber. 1992. Mineralocorticoid excess, dietary sodium and myocardial fibrosis. J. Lab. Clin. Med. 120:893-901.

20. Young, M., M. Fullerton, R. Dilley, and J. Funder. 1994. Mineralocorti- coids, hypertension and cardiac fibrosis. J. Clin. Invest. 93:2578-2583.

21. Cowley, A.W. 1992. Long-term control of arterial blood pressure. Physiol. Rev. 72:231-278

22. Onoyama, K., E.L. Bravo, and R.C. Tarazi. 1979. Sodium, extracellular fluid volume, and cardiac output changes in the genesis of mineralocorticoid hypertension in the intact dog. Hypertension (Dallas). 1:331-336.

23. Pan, Y., and D.B. Young. 1982. Experimental aldosterone hypertension in the dog. Hypertension (Dallas). 4:279-287.

24. Daniels, B.S., and T.H. Hostetter. 1990. Adverse effects of growth in the glomerular circulation. Am. J. Physiol. 258:F1409-F1416.

25. Lax, D.S., J.A. Benstein, E. Tolbert, and L.D. Dworkin. 1992. Effects of salt restriction on renal growth and glomerular injury in rats with remnant kidneys. Kidney Int. 41:1527-1534.

26. Smith, J.M., and A.W. Jones. 1990. Calcium antagonists inhibit elevated potassium efflux from aorta of aldosterone-salt hypertensive rats. Hypertension (Dallas). 15:78-83.

27. Wang, W. 1994. Chronic administration of aldosterone depresses baroreceptor reflex function in the dog. Hypertension (Dallas). 24:571-575.

28. Lariviere, R., G. Thibault, and E.L. Schiffrin. 1993. Increased endothelin-1 content in blood vessels of deoxycorticosterone acetate- - salt hypertensive but not in spontaneously hypertensive rats. Hypertension (Dallas). 21:294-300.

29. Ullian, M.E., J.R. Schelling, and S.L. Linas. 1992. Aldosterone enhances angiotensin II receptor binding and inositol phosphate responses. Hypertension (Dallas). 20:67-73.

30. Dworkin, L.D., T.H. Hostetter, H.G. Rennke, and B.M. Brenner. 1984. Hemodynamic basis for glomerular injury in rats with desoxycorticosterone salt hypertension. J. Clin. Invest. 73:1448-1461.

31. Wakisaka, M., M.J. Spiro, and R.G. Spiro. 1994. Synthesis of type VI collagen by cultured glomerular cells and comparison of its regulation by glucose and other factors with that of type IV collagen. Diabetes. 43:95-103.

32. Todd-Turla, K.M., J. Schnermann, G. Fejes-Toth, A. Naray-Fejes-Toth, A. Smart, P.D. Killen, and J.P. Briggs. 1993. Distribution of mineralocorticoid and glucocorticoid receptors mRNA along the nephron. Am. J. Physiol. 264: F781-F791.

33. Wehling, M., M. Christ, and K. Theisen. 1992. Membrane receptors for aldosterone: a novel pathway for mineralocorticoid action. Am. J. Physiol. 263 : E974-E979.

34. Wehling, M., J. Kasmayr, and K. Tikerson. 1991. Rapid effects of mineralocorticoids on sodium-proton exchanger: genomic or nongenomic pathway? Am. J. Physiol 260 (Endocrine Metab 23):E719-E726. 